\title{
Norois
}

Environnement, aménagement, société

$208 \mid 2008 / 3$

Gestion du littoral, risques urbains, ours en Pyrénées

\section{Évolution du trait de côte à Nouakchott (Mauritanie) de 1954 à 2005 par photo- interprétation}

Aerial photo-interpretation of shoreline changes at Nouakchott (Mauritania)

from 1954 to 2005

Ibrahima B. Nd. Faye , Alain Hénaff, Françoise Gourmelon et Amadou

Tahirou Diaw

\section{OpenEdition \\ Journals}

Édition électronique

URL : https://journals.openedition.org/norois/2146

DOI : $10.4000 /$ norois. 2146

ISBN : 978-2-7535-1556-7

ISSN : 1760-8546

Éditeur

Presses universitaires de Rennes

Édition imprimée

Date de publication : 1 novembre 2008

Pagination : 11-27

ISBN : 978-2-7535-0788-3

ISSN : 0029-182X

Référence électronique

Ibrahima B. Nd. Faye, Alain Hénaff, Françoise Gourmelon et Amadou Tahirou Diaw, «Évolution du trait de côte à Nouakchott (Mauritanie) de 1954 à 2005 par photo-interprétation », Norois [En ligne], 208 | 2008/3, mis en ligne le 01 novembre 2010, consulté le 15 février 2023. URL : http:// journals.openedition.org/norois/2146 ; DOI : https://doi.org/10.4000/norois.2146

\section{c) (†) $\odot$}

Creative Commons - Attribution - Pas d'Utilisation Commerciale - Pas de Modification 4.0 International - CC BY-NC-ND 4.0

https://creativecommons.org/licenses/by-nc-nd/4.0/ 


\title{
ÉVOLUTION DU TRAIT DE CÔTE À NOUAKCHOTT (MAURITANIE) DE 1954 À 2005 PAR PHOTO-INTERPRÉTATION
}

\author{
Ibrahima B. Nd. FaYe ${ }^{1}$, Alain HenafF ${ }^{1}$, \\ Françoise Gourmelon ${ }^{1}$, Amadou Tahirou DiaW ${ }^{2}$ \\ ${ }^{1}$ GEOMER - UMR 6554 LETG CNRS \\ (Université de Bretagne Occidentale), \\ IUEM, Technopôle Brest-Iroise, place Nicolas Copernic - 29280 Plouzané, France \\ ibrahima.faye@univ-brest.fr,alain.henaff@univ-brest.fr,françoise.gourmelon@univ-brest.fr \\ ${ }^{2}$ Laboratoire d'Enseignement et de Recherche en Géomatique (LERG) \\ Campus universitaire de l'ESP - BP 25275 DAKAR-FANN, Sénégal \\ atdiaw@ucad.sn
}

\begin{abstract}
RÉSUMÉ
Cette étude présente une analyse diachronique de l'évolution de la ligne de rivage aux abords de Nouakchott (1954-2005) par photo-interprétation. Elle utilise comme références les lignes des pleines mers et du pied de dune. Les variations observées de la position du pied de dune entre 1954 et 1980 témoignent d'un littoral en érosion à un rythme moyen de -0,83 m/an. L'étude de la cinématique de la ligne des pleines mers entre 1980 et 2005 indique des évolutions importantes consécutives à l'aménagement de la jetée du port de Nouakchott en 1986. Le trait de côte a progressé de plus de $800 \mathrm{~m}$ en amont-dérive alors qu'au sud de l'épi, en aval-dérive, la côte a reculé de plus de $500 \mathrm{~m}$.

Les principales limites de l'approche méthodologique de cartographie de la mobilité du trait de côte résident dans les erreurs d'interprétation des lignes de référence; celles-ci sont plus spécifiquement liées ici à la qualité des images aériennes utilisées et aux variations à court terme de la position de la limite des pleines mers. Ce sont là autant de sources d'erreur qu'il faut analyser et estimer avant de tirer des conclusions sur la cinématique littorale.
\end{abstract}

MotS CLÉ : trait de côte - ligne des pleines mers - pied de dune - Nouakchott - photographies aériennes - érosion - accrétion

\section{ABSTRACT \\ Aerial photo-interpretation of shoreline changes at Nouakchott (Mauritania) from 1954 to 2005}

This paper presents a diachronic analysis of shoreline changes (1954-2005) around Nouakchott by interpretation of aerial photographs. Two shoreline indicators are used: the dune foot and the high water line. The comparison of the dune foot location between 1954 and 1980 shows a coastline in erosion with an average rate of - $-8,83$ m/year. The study of the high water line kinematics from 1980 to 2005 points out large evolutions consecutive to the construction of the harbour jetty in 1986. Thus the shoreline has progressed more 
than $800 m$ in the updrift while in the downdrift side (South of the groin) it has retreated over half kilometer.

The main limits of the method are the misinterpretation of the reference lines due to the poor quality of some aerial photographs and the short-term variability in the high water line position. All that may lead to errors that must be analysed and assessed before drawing conclusions on coastal evolution.

KEY WORDS : shoreline - coastline - high water line - dune foot-Nouakchott-aerial photographs - coastal erosion - accretion

Le processus de recul du trait de côte affecte actuellement plus de $70 \%$ des plages de la planète (Bird, 1985). Les littoraux ouest-africains constitués en grande partie d'estuaires et de plages n'échappent pas à cette évolution. Les taux d'érosion estimés par A. C. Ibe et R. E. Quelennec (1989) varient de 1,2 à 6 m/an sur les secteurs sableux du Sénégal à la Sierra Léone. Or l'essentiel de la population et des activités économiques est concentré dans cette région sur les zones côtières. En effet, toutes les grandes agglomérations ouest-africaines (Nouakchott, Saint-Louis, Dakar, Banjul, Bissau, Conakry, Freetown, Monrovia, etc.) sont localisées sur la côte, foyer privilégié d'immigration et de développement de l'économie maritime : port, pêche, industries, tourisme balnéaire, etc. (Quensière et al., 2006). Le littoral ouest-africain abrite également des aires protégées reconnues mondialement telles que le banc d'Arguin, le parc national des Oiseaux de Djoudj, le delta du Saloum, l'Archipel des Bijagos, etc. que les changements littoraux pourraient affecter.

Dans ce contexte, l'étude de la dynamique des littoraux sableux d'Afrique de l'Ouest a été entreprise au sein des activités scientifiques du Programme Régional de Conservation des Zones Côtières et Marines en Afrique de l'Ouest (PRCM). Elle est fondée, d'une part, sur une synthèse régionale et, d'autre part, sur l'étude à grande échelle de cas représentatifs de la diversité des situations rencontrées sur les côtes sableuses : plages associées à des cordons dunaires isolant des dépressions interdunaires, plages sans cordon dunaire, plages associées à des flèches sableuses, plages associées à des cordons littoraux isolant des mangroves, etc. Parmi les sites retenus dans les différents pays de la sous région (Mauritanie, Sénégal, Gambie, Guinée Bissau), les abords de Nouakchott en Mauritanie sont exemplaires (fig. 1). Nouakchott, capitale depuis 1957 a connu une importante croissance démographique en une cinquantaine d'années. De moins de 5000 habitants en 1959, elle en abrite actuellement plus de 700000 . Cette situation a conduit à la multiplication d'activités anthropiques sur le littoral : constructions, prélèvement de bois de chauffe et extraction de matériaux sur le cordon dunaire, aménagement d'infrastructures portuaires responsables d'importantes modifications du trait de côte (Marico, 1996; Philippon, 1999; Ould El Moustapha, 2000).

Démarrés en avril 1979, les travaux de construction du port de Nouakchott se sont achevés en juillet 1986. Selon le rapport de Z. Bai et al. (1997), les changements dans la position du trait de côte et la bathymétrie des petits fonds ont commencé après la construction en 1983 du premier tronçon de brise-lames. Cet ouvrage, accolé au quai et destiné à atténuer l'énergie des vagues, a provoqué rapidement un engraissement au droit du brise-lames et un ensablement du bassin. Pour bloquer le transit sédimentaire et éviter la sédimentation dans le bassin, un mur de 496 m de long a été construit en 1986 sous la passerelle (fig. 1). Le blocage de la dérive littorale s'est traduit au nord par une avancée et au sud par un recul très rapides de la ligne de rivage.

Ces processus ont été étudiés par de nombreux auteurs qui ont estimé les changements intervenus au voisinage immédiat du port depuis les années 1980 jusqu'en 2002 (Ould El Ghassem, 1987; Azandossessi, 1988; Ould Eleya, 1994; Marico, 1996; Ould El Moustapha, 2000; Wu, 2003 ; GRESARC, 2006 ; Ould El Moustapha et al., 2007). Les documents, supports de ces études, sont de nature et d'échelle spatiale diverses : cartes topographiques, images satellitaires, pho- 
Figure 1 : Localisation et morphologie de la zone d'étude

Location and morphology of the study area

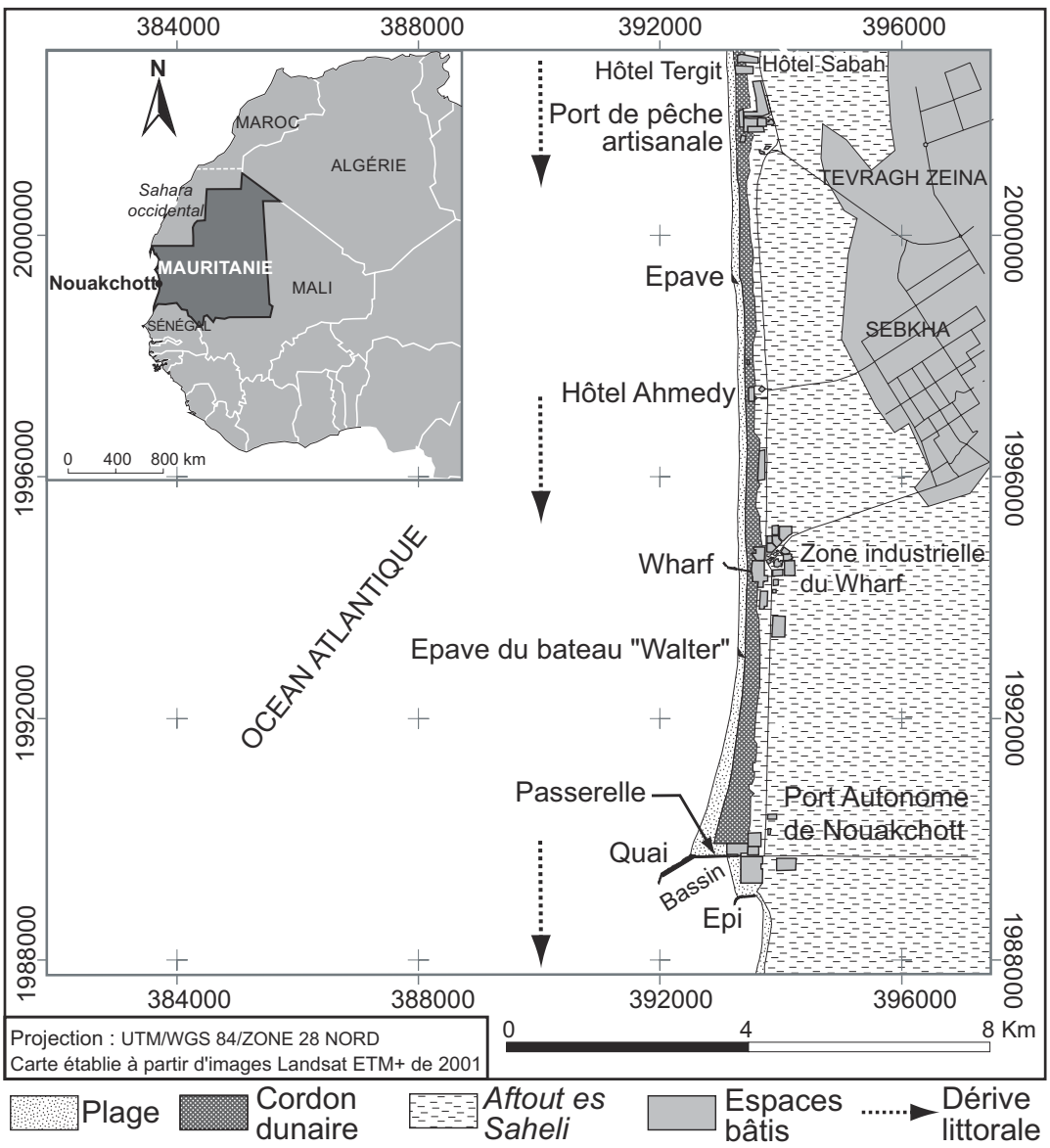

tographies aériennes, levés GPS et DGPS. Peu d'entre elles indiquent la ligne de référence utilisée pour le suivi du trait de côte à l'exception de deux études récentes. Wu (2003) utilise la ligne de rivage instantanée alors que le GRESARC (2006) utilise la ligne des basses mers. Les méthodes mises en ouvre pour suivre l'évolution de la position du trait de côte sont les suivantes :

- mesures linéaires sur des photographies aériennes non rectifiées (Azandossessi, 1988 ; Marico, 1996);

- estimation de surfaces en accrétion ou en érosion à partir d'images satellitaires SPOT redressées (Ould Eleya, 1994; Wu, 2003);

- levés DGPS et modélisation de l'évolution future de la ligne de rivage (Ould El Moustapha, 2000 ; GRESARC, 2006 ; Ould El Moustapha et al., 2007).

La diversité des pas de temps considérés, celle des échelles spatiales et des méthodes utilisées dans ces travaux aboutissent à une forte hétérogénéité des valeurs de recul ou d'avancée du trait de côte. En règle générale, les taux d'évolution proposés varient entre -20 et $-35 \mathrm{~m} / \mathrm{an}$ au sud du port et entre +22 et $+42 \mathrm{~m} / \mathrm{an}$ au nord. 


\begin{tabular}{|c|c|c|c|c|}
\hline \multirow{2}{*}{ Source de données } & \multirow{2}{*}{ Ligne de référence } & \multicolumn{2}{|c|}{ Taux d'évolution moyen } & \multirow{2}{*}{ Auteurs } \\
\hline & & au nord du port & au sud du port & \\
\hline \multicolumn{5}{|c|}{ Mobilité du trait de côte } \\
\hline $\begin{array}{c}\text { Photographies aériennes } \\
\text { obliques (1986) }\end{array}$ & ? & ? & $-35 \mathrm{~m} / \mathrm{an}$ & Azandossessi, 1988 \\
\hline $\begin{array}{l}\text { Photographies aériennes } \\
\text { IGN }(1980,1991) \text { et des } \\
\text { mesures de terrain (1993) }\end{array}$ & $?$ & $+42,5 \mathrm{~m} / \mathrm{an}$ & $-20,45 \mathrm{~m} / \mathrm{an}$ & Marico, 1996 \\
\hline $\begin{array}{c}\text { Carte topographique IGN } \\
\text { (1980), images satellitaires } \\
(1987,1989,1992) \text {, levés } \\
\text { DGPS (1998) }\end{array}$ & ? & $+35 \mathrm{~m} / \mathrm{an}$ & $-25 \mathrm{~m} / \mathrm{an}$ & $\begin{array}{l}\text { Ould El Moustapha, } \\
2000\end{array}$ \\
\hline $\begin{array}{c}\text { Levés GPS (1995), levés } \\
\text { DGPS (2006) }\end{array}$ & Ligne des basses mers & $+22 \mathrm{~m} / \mathrm{an}$ & $-28 \mathrm{~m} / \mathrm{an}$ & GRESARC, 2006 \\
\hline $\begin{array}{c}\text { Carte topographique IGN } \\
\text { (1980), images SPOT (1987, } \\
\text { 1992), levés DGPS (1998, } \\
\text { 2002) }\end{array}$ & ? & $+32,7 \mathrm{~m} / \mathrm{an}$ & $-25 \mathrm{~m} / \mathrm{an}$ & $\begin{array}{c}\text { Ould El Moustapha } \\
\text { et al., } 2007\end{array}$ \\
\hline \multicolumn{5}{|c|}{ Surfaces accumulées ou érodées } \\
\hline $\begin{array}{c}\text { Images satellitaires rectifiées } \\
(1987,1988,1989,1991, \\
1992)\end{array}$ & ? & $+0,0862 \mathrm{~km}^{2} / \mathrm{an}$ & $-0,0974 \mathrm{~km}^{2} / \mathrm{an}$ & Ould Eleya, 1994 \\
\hline $\begin{array}{c}\text { Images SPOT rectifiées XS2 } \\
(1989) \text { et Pan }(1995,1999, \\
2001)\end{array}$ & $\begin{array}{l}\text { Ligne instantanée de } \\
\text { rivage }\end{array}$ & $+0,08 \mathrm{~km}^{2} / \mathrm{an}$ & $-0,12 \mathrm{~km}^{2} / \mathrm{an}$ & Wu, 2003 \\
\hline
\end{tabular}

Tableau 1 : Quelques sources de données et taux d'évolution du trait de côte estimés aux abords du port de Nouakchott Some sources of data and shoreline change rates assessed around Nouakchott harbour

La seule étude qui s'est intéressée à la mobilité du trait de côte avant l'implantation du port de Nouakchott est celle de J.-P. Barusseau (1985). La méthode utilisée était basée sur des mesures de distances entre la ligne de swash et des points repérés sur des clichés aériens non rectifiés datant de 1954 et de 1980. Une analyse qualitative en a été déduite permettant de conclure à un littoral en érosion dont la vitesse moyenne annuelle de recul était certainement inférieure à $1 \mathrm{~m}$. L'objectif de notre étude est de faire le point sur les évolutions du linéaire côtier de Nouakchott à une échelle mi-séculaire en s'appuyant sur des photographies aériennes de 1954, 1980 et 2005. La méthode utilisée est décrite et les principaux résultats commentés en tenant compte des incertitudes inhérentes à la démarche.

\section{Cadre physique actuel du secteur étudié}

Situé dans la partie centrale de la « grande plage mauritanienne » (De Lanjamet, 1988), le littoral de Nouakchott présente un aspect morphologique relativement simple. Il est formé par une plage de sable blanc coquillier associée à un cordon dunaire ensellé barrant une dépression de type sebkha, l'Aftout es Saheli (fig. 1). C'est une côte ouverte sur le large, caractérisée par un puissant déferlement lié à la présence de barres prélittorales (De Lanjamet, 1988). Les houles dominantes proviennent essentiellement des secteurs nord-ouest, ouest nord-ouest et ouest avec des périodes de 8 à 11 secondes et des hauteurs significatives de 1,64 à 2,26 m (Ould El Moustapha et al., 2007). Le volume de la charge sédimentaire charrié par la dérive littorale orientée du nord au sud est estimé entre 875000 et $1100000 \mathrm{~m}^{3} / \mathrm{an}$. Ce transit sédimentaire constitue avec le vent les principaux agents naturels de la dynamique littorale (Ould El Moustapha, 2000). Le marnage moyen annuel est de 0,93 m (Bai et al., 1997). Concernant les conditions anémométriques, l'ana- 
lyse des vents dominants de 1960 à 2004 à la station de Nouakchott montre qu'ils proviennent essentiellement du secteur nord (nord-est à nord-ouest) avec une prépondérance des composantes nord nord-ouest et nord-ouest. La répartition intermensuelle des vents dominants indique trois saisons dans l'année. Ainsi de décembre à février, le vent dominant est de secteur nord-est. Il passe au nord-ouest et nord nord-ouest entre mars et juin, alors que de juillet à septembre, les flux dominants viennent de l'ouest. La vitesse moyenne de ces alizés est comprise entre 4,4 et 4,7 m/s. D'après les études de D. Marico (1996) et S. Philippon (1999), ces vents sont d'un point de vue théorique suffisamment efficaces pour générer un transit éolien, et ce, d'autant plus que le couvert végétal est très épars voire inexistant.

La zone couverte par cette étude s'étend sur environ $13 \mathrm{~km}$ de côte du sud du port de pêche artisanale jusqu'au sud du port autonome de Nouakchott (fig. 1). Actuellement la morphologie et l'orientation du trait de côte sont caractéristiques de trois secteurs.

- Entre le port de pêche et le wharf, le trait de côte a une orientation nord sud. La plage de 30 à $50 \mathrm{~m}$ de large est bordée par un cordon de dunes vives parsemé de touffes de Zygophyllum waterlotii, Tamarix sp., et de Nitraria retusa (largeur : 150 à 200 m) (photo 1).

Photo 1 : Le cordon dunaire parsemé de touffes de végétation et d'étendue modeste ( 150 à $200 \mathrm{~m}$ de large) au nord du wharf, vue prise en direction du nord, le 22 août 2007

\section{Narrow}

coastal dune (150 to

$200 \mathrm{~m}$ wide) with scattered vegetation in the north of the wharf, photo taken towards the north, august 22, 2007

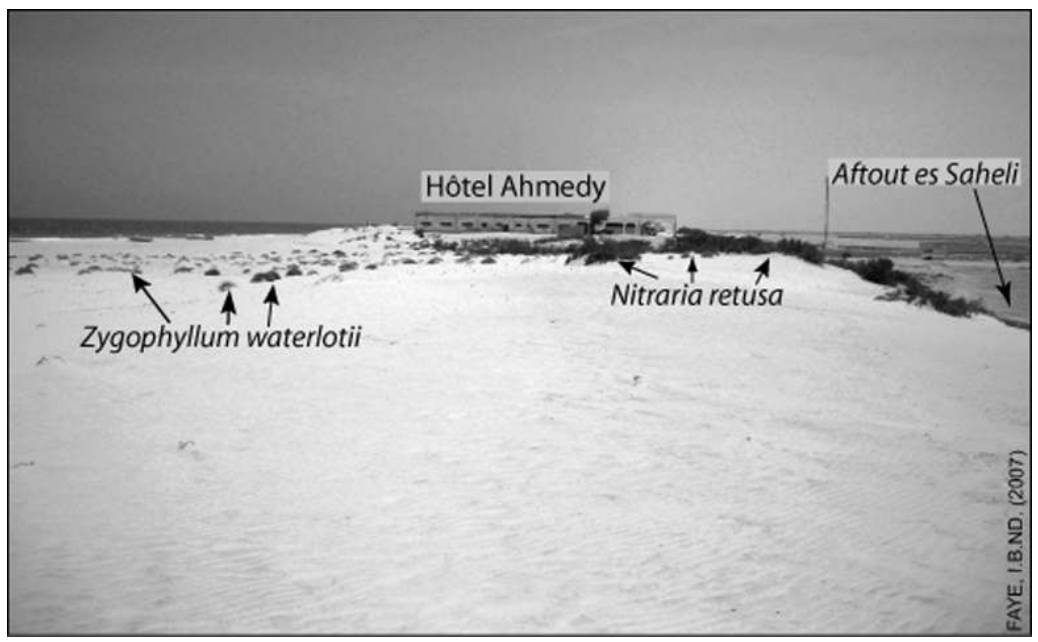

- Au sud du wharf, l'épave du bateau Walter marque un net changement d'orientation du trait de côte qui prend une direction nord nord-est sud sud-ouest. L'accumulation des sédiments au nord de la digue du port est responsable de cette incurvation. C'est dans cette zone que le cordon dunaire tapissé de Zygophyllum waterlotii et de Nitraria retusa atteint sa largeur maximale. De $300 \mathrm{~m}$ aux environs de l'épave du bateau Walter, il s'étend sur plus de $800 \mathrm{~m}$ de large au niveau de la passerelle du port (photo 2).

- Au sud des installations du port autonome, l'érosion accélérée par l'épi et la jetée sous la passerelle, donne à la côte un plan incurvé. L'estran, qui suit une direction nord nord-ouest sud sud-est après le quai, s'oriente progressivement vers le nord nord-est sud sud-ouest en aval de l'épi. Dans ce secteur, le cordon dunaire a disparu favorisant ainsi un contact direct de la plage avec les sebkhas de l'Aftout es Saheli (photo 3).

\section{Méthodologie}

La méthodologie utilisée dans cette étude repose sur des techniques de photo-interprétation assistée par ordinateur décrites par de nombreux auteurs (Shoshany et Degani, 1992; Durand, 2000 ; Moore, 2000; Dehouck, 2006). L’approche comprend les étapes suivantes : 

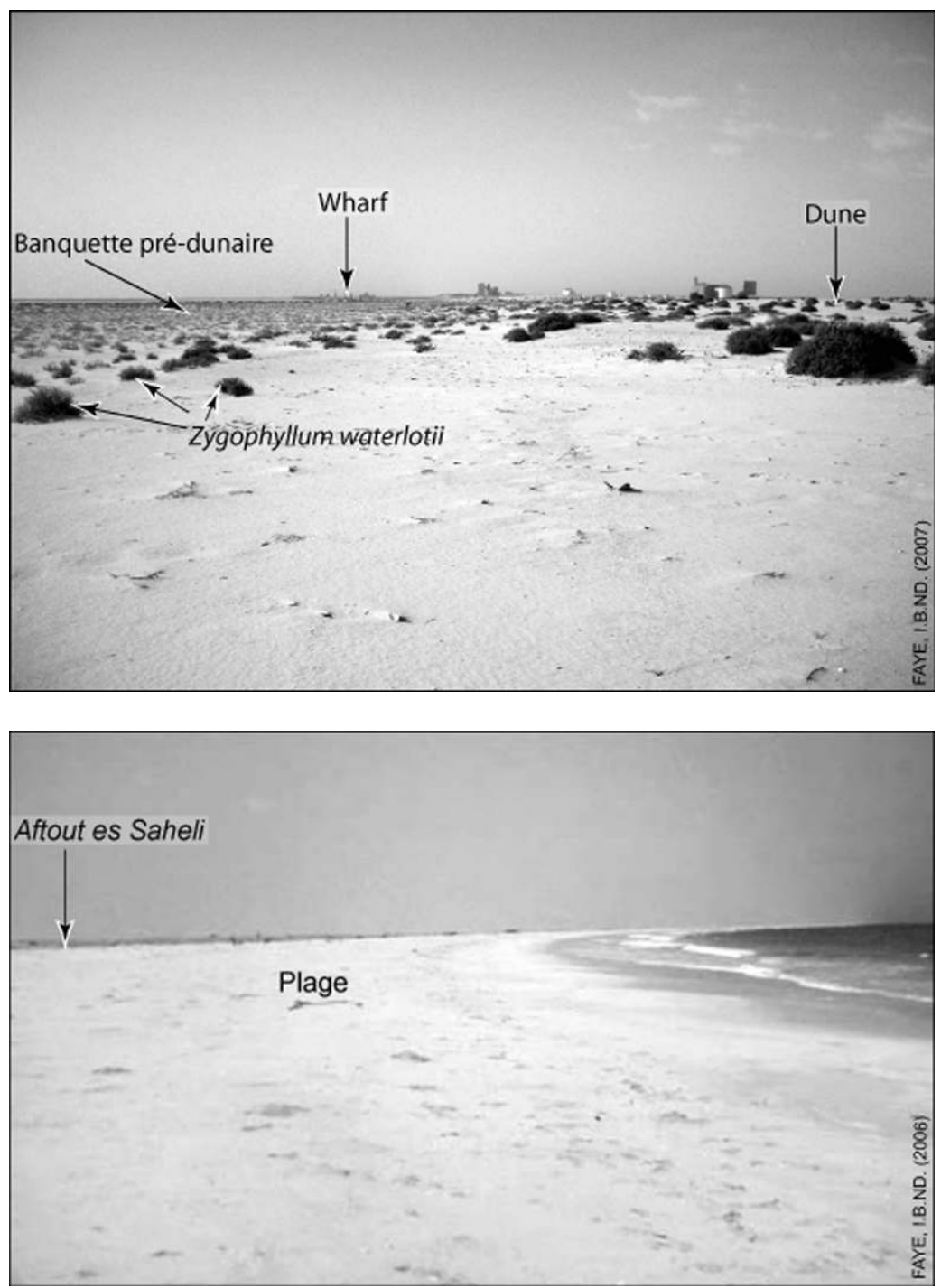

Photo 2 : Aperçu du cordon dunaire au sud du wharf

En avant de la dune préexistante, on observe une banquette prédunaire couverte de Zygophyllum waterlotii, résultat de l'accumulation des sédiments au nord de la jetée du port, vue prise en direction du nord, le 16 août 2007.

\section{Coastal dumes in the south of the wharf \\ A grassy foredune has developed in front of the pre-existent dune, it's the result of shore- line accretion in the north of harbour jetty, photo taken towards the north, angust 16, 2007.}

Photo 3 : Absence du cordon dunaire au sud du port de Nouakchott Un placage sableux sépare la plage de l'Aftout es Saheli, vue prise en direction du sud, le 03 août 2006.

Disappearence of the dune in the south of Nouakchott harbour A sandy surface separates the beach from Aftout es Saheli, photo taken towards the south, august 3, 2006.

- choix d'une ligne de référence;

- rectification géométrique des images aériennes disponibles;

- digitalisation du trait de côte et estimation des erreurs;

- étude de la cinématique du trait de côte.

\section{LE CHOIX D'UNE LIGNE DE RÉFÉRENCE}

Il existe plus d'une douzaine de lignes de référence matérialisant la position du trait de côte (Robin, 2002; Boak et Turner, 2005). Dans le cadre d'une étude diachronique fondée sur la photointerprétation, le choix et l'extraction d'une ligne commune aux différentes images utilisées est nécessaire. Sur les images de 1954, deux lignes sont identifiables : la ligne de rivage instantanée et le pied de dune qui représente la limite du front dunaire, matérialisé par une rupture de pente en avant de la limite de végétation dunaire (photo 4). En plus de ces deux lignes, sur les images 
de 1980, le niveau des pleines mers matérialisé par la limite supérieure d’humectation peut être également individualisé (photo 5). Il indique le niveau atteint sur l'estran par la marée haute pendant toute la période de l'étale (à ce titre, cette limite est bien plus représentative que la ligne de rivage instantanée qui ne se réfère qu'à une seule vague). Sur les images de 2005 enfin, seules la ligne de rivage instantanée et celle correspondant aux pleines mers sont discernables; le contact entre la plage et la dune est difficilement exploitable en l'absence d'une nette rupture de pente, résultat de l'étalement du cordon dunaire.

En définitive, la ligne de rivage instantanée est la seule limite de référence présente sur toutes les images. Elle n'est cependant pas utilisable ici car étant dépendante des conditions de marée, elle reste fluctuante d'une date à l'autre. Or, aucune information n'est disponible sur les jours et les heures des prises de vue, et les hauteurs des marées correspondantes pour pouvoir effectuer les corrections nécessaires. À partir de là, deux lignes de référence ont été retenues : pour la période 1954-1980, le pied de dune a été utilisé pour positionner et mesurer l'évolution du trait de côte; pour la période 1980-2005, le choix s'est porté sur la ligne des pleines mers précédant la prise de vue. Toutefois, comme pour la ligne de rivage instantanée, cette limite reste également dépendante de la marée. Nous verrons néanmoins dans un paragraphe ultérieur comment la méthode que nous avons mise en œuvre a permis d'évaluer les différences de hauteurs d'eau entre une pleine mer de vive-eau et de morte-eau, et par là même, d'estimer les marges d'erreur en matière de positionnement du trait de côte.

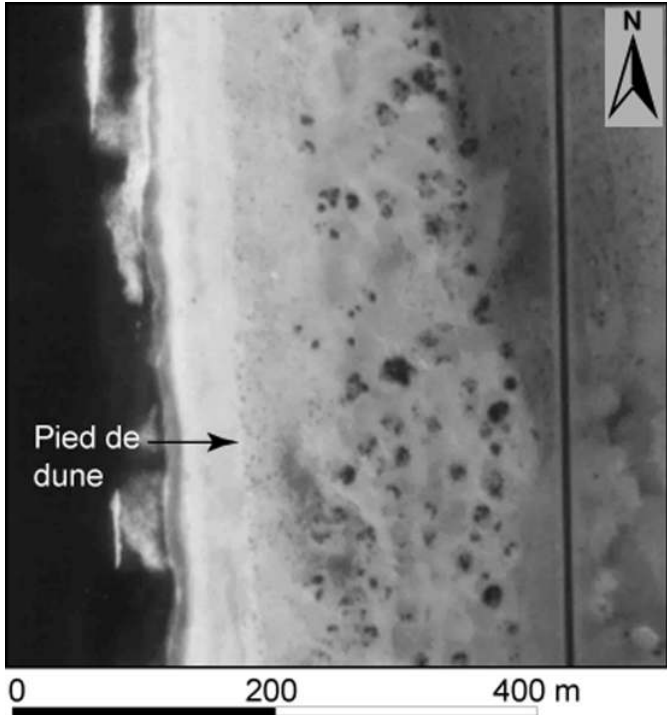

Photo 4 : Pied de dune (extrait d'une photographie aérienne, IGN 80 MAU-4-215, 1980)

Aerial view of the dune foot derived from aerial photograph (IGN 80 MAU-4-215, 1980)

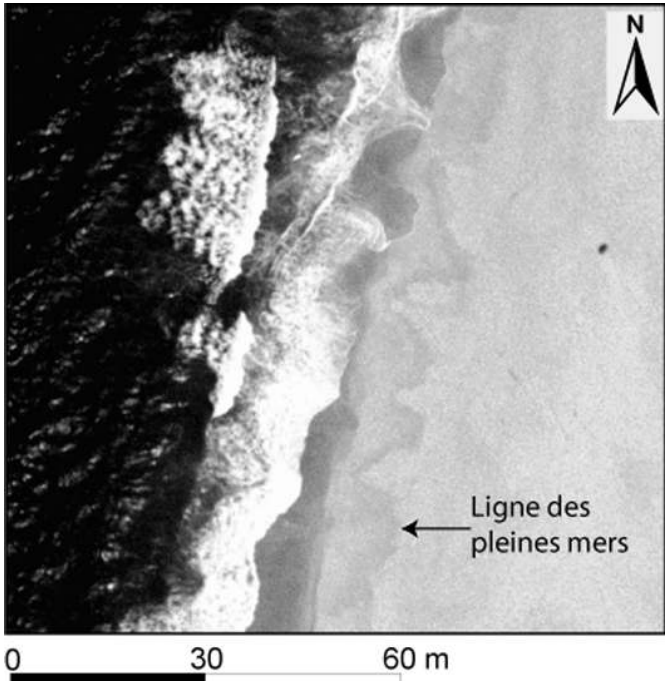

Photo 5 : Ligne des pleines mers (extrait d'une photographie aérienne, Société Hauts Monts, MR 05-01, 2005)

Aerial view of the high water line derived from aerial photograph (Société Hauts Monts, MR 05-01, 2005)

\section{RECTIFICATION GÉOMÉTRIQUE DES IMAGES AÉRIENNES DISPONIBLES}

Les informations sur la position de la ligne de rivage proviennent des clichés de trois missions aériennes, acquis en mode panchromatique en 1954, 1980 et 2005 (tableau 2). Ces clichés nous ont été fournis au format numérique par les responsables du Programme Régional de Conservation de la Zone Côtière et Marine en Afrique de l'Ouest (PRCM). 


\begin{tabular}{|c|c|c|c|c|}
\hline Année & Références de la mission & Échelle des clichés & $\mathrm{N}^{\circ}$ des clichés utilisés & Taille du pixel (m) \\
\hline 1954 & IGN AOF 1954-096 & $1 / 50000$ & $072 ; 068$ & 2,11 \\
\hline 1980 & IGN 80 MAU-4-215 & $1 / 21500$ & $5 ; 7 ; 9 ; 11$ & 0,91 \\
\hline 2005 & Société Hauts Monts, MR 05-01 & $1 / 15000$ & $\mathrm{~B} 1-12 ; \mathrm{B} 1-15 ; \mathrm{B} 1-16$ & 0,33 \\
\hline
\end{tabular}

Tableau 2 : Missions photographiques utilisées pour l'analyse diachronique de la cinématique du trait de côte aux abords de Nouakchott of Nouakchott

Aerial photographic missions used for diachronic analysis of shoreline changes at the neighbourhoods

Ces images ont été rectifiées géométriquement et projetées dans le système de projection cartographique UTM/WGS 84/ZONE 28 Nord. Les coordonnées de points d'amers repérés sur les images de 2005 ont été collectées sur le terrain au moyen d'un GPS (MLR ${ }^{\circledR}$, modèle SP24 XC). La méthode polynomiale classiquement utilisée pour rectifier des cartes (Thieler et Danforth, 1994a) et des images aériennes (Shoshany et Degani, 1992; Durand, 1998, 2000) a été retenue. Le logiciel ENVI 4.2 a été utilisé pour effectuer la rectification avec un polynôme de second degré, 14 à 18 points de calage par image et une résolution spatiale de $1 \mathrm{~m}$. Cette résolution correspond à la moyenne de la taille des pixels des images originales. Les images de 2005 redressées ont servi de référence pour le calage image à image des images antérieures. Puis de manière à valider les corrections géométriques, les images multidates d'un site ont été superposées à l'écran pour vérifier la superposition des éléments remarquables et des infrastructures présentes sur le cordon dunaire ou à proximité (route, wharf, bâtiments, etc.). En cas de mauvais résultats, l'image subit une rotation, une mise à l'échelle et une translation afin d'obtenir une superposition satisfaisante des clichés pour les assembler et extraire le trait de côte.

\section{DigitALISATION DU TRAIT DE CÔTE ET ESTIMATION DES ERREURS}

La digitalisation a été réalisée avec le logiciel ArcView 3.2a sur fond d’images redressées (1954, 1980 et 2005). En dépit du redressement des images aériennes et de l'automatisation des mesures, il demeure un certain nombre d'incertitudes liées à la qualité des données de base, à la rectification des images, à l'extraction du trait de côte et à la variation à court terme de la position de la ligne de référence. Dans le cadre de synthèses diachroniques, tous ces éléments sont des facteurs de décalages supplémentaires qu'il convient d'évaluer avant de tirer des conclusions sur la cinématique littorale (Anders et Byrnes, 1991 ; Crowell et al., 1991; Thieler et Danforth, 1994a; Durand, 1998, 2000; Gaillot et Chaverot, 2001 ; Robin, 2002).

\section{Erreurs liées à la collecte des amers sur le terrain}

Cette incertitude est déterminée par la précision du GPS utilisé pour collecter les points de calage sur le terrain. Selon les spécifications techniques du fabriquant, le modèle utilisé est précis à $3 \mathrm{~m}$ RMS en mode $2 \mathrm{D}(\mathrm{X}, \mathrm{Y})$. Pour valider la précision communiquée par le fabriquant, nous avons comparé les mesures GPS avec les coordonnées de trois bornes géodésiques de l'IGN. Sur 363 mesures effectuées au GPS au niveau de ces points géodésiques, l'écart moyen quadratique (moyenne quadratique de la somme des carrés des écarts en x et y entre chaque position enregistrée par le GPS et les coordonnées des points géodésiques) est de 3,92 m. La précision de la localisation dépend de la position et du nombre de satellites disponibles au moment de l'enregistrement, du temps de mesure et de la présence d'obstacles aux alentours susceptibles de perturber la trajectoire ainsi que l'acquisition du signal. Afin de minimiser cette erreur, toutes nos mesures ont été acquises en présence d'au moins dix satellites et les amers sélectionnés sont localisés préférentiellement dans des zones dégagées. Nous pouvons donc estimer l'incertitude liée à la position du GPS à environ $4 \mathrm{~m}$. 


\section{Erreurs liées au géoréférencement des images aériennes}

Le géoréférencement correct d'une image nécessite un nombre suffisant d'amers bien répartis sur l'ensemble de la zone d'étude. Or sur les images de base utilisées (2005), il n’a pas été aisé de réunir ces conditions. Elles offrent en effet une très bonne capacité d'identification de la ligne de référence mais la possibilité de choisir un grand nombre d'amers est réduite car la zone terrestre couverte, peu occupée par ailleurs, n'excède pas $2 \mathrm{~km}$. Une autre contrainte notable est la concentration des amers potentiels autour du Wharf et du port. Pour pallier ce problème, en plus des amers classiques (coins de bâtiments, murs, croisement de routes, etc.), nous avons dû utiliser comme amers des touffes de végétation notamment de Nitraria retusa (photo 6) et de Tamarix sp. Cependant, dans certaines parties des clichés de 1954, nous n'avons pas pu retrouver suffisamment de touffes bien réparties pour redresser convenablement les images. Lors de la vérification du géoréférencement, des décalages de 5 à $7 \mathrm{~m}$ sont apparus localement. Ces secteurs, représentant 580 m de linéaire côtier, ont été exclus de l'étude.

Photo 6 : Touffe de Nitraria retusa utilisée comme amer sur les images aériennes de 2005 (Mission MR 05-01, 2005, Société Hauts Monts, 2005)

\section{Tuft of Nitraria} retusa used as a ground control point on aerial image of 2005 (MR 05-01 mission, Société Hauts Monts, 2005)

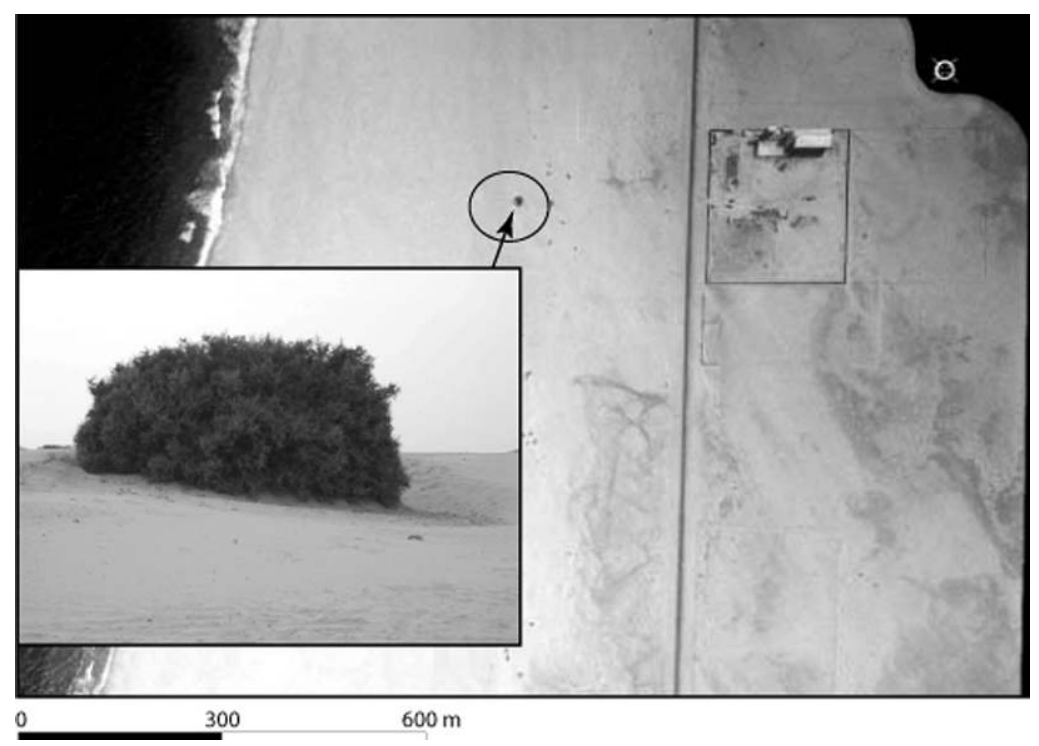

Généralement, les erreurs RMS pour chaque image étaient inférieures ou égales à 0,5 pixel soit $0,5 \mathrm{~m}$ (tableau 3). Toutefois, P. Durand (2000) estime qu'il faut ramener cette erreur à \pm 1 pixel soit 2 pixels si on compare deux images.

\section{Erreurs liées à la digitalisation du trait de côte}

La méthode utilisée pour extraire la limite supérieure d’humectation de l'estran et le pied de dune est fondée sur la photo-interprétation assistée par ordinateur. En fonction des variations de ton sur l'image, la ligne est digitalisée. La précision de la ligne ainsi extraite dépend de l'expérience du photo-interprète, de son appréciation de la ligne de référence elle-même conditionnée par la résolution, l'échelle et la qualité radiométrique des images. Par conséquent, le trait de côte acquis par cette méthode est approximatif. Dans le but de réduire la portée de cette incertitude sur les taux d'évolution du trait de côte, les zones où les lignes de référence étaient trop ambiguës à cause d'un mauvais contraste de l'image n'ont pas fait l'objet de mesures. Ce sont en général des petits segments de 10 à une centaine de mètres de long. Ils représentent un total de $605 \mathrm{~m}$ pour le pied de dune, $2261 \mathrm{~m}$ pour la ligne des pleines mers et concernent essentiellement les clichés de 1980. En effet, alors que sur la mission de 1954 on observe un front dunaire continu sur l'ensemble des 


\begin{tabular}{|c|c|c|c|}
\hline Date des prises de vues & $\mathrm{N}^{\circ}$ des images rectifiées & Nombre de points de calage & Erreur RMS (m) \\
\hline \multirow{2}{*}{1954} & 068 & 14 & 0,53 \\
\hline & 072 & 15 & 0,58 \\
\hline \multicolumn{3}{|c|}{ Moyenne } & 0,55 \\
\hline \multirow{4}{*}{1980} & 11 & 14 & 0,30 \\
\hline & 9 & 14 & 0,24 \\
\hline & 7 & 18 & 0,29 \\
\hline & 5 & 15 & 0,35 \\
\hline \multicolumn{3}{|c|}{ Moyenne } & 0,29 \\
\hline \multirow{3}{*}{2005} & B1-12 & 18 & 0,23 \\
\hline & B1-15 & 16 & 0,21 \\
\hline & B1-16 & 17 & 0,16 \\
\hline \multicolumn{3}{|c|}{ Moyenne } & 0,20 \\
\hline
\end{tabular}

Tableau 3 : Erreurs RMS liées à la rectification des images

RMS errors linked to the image registration

images, la mission de 1980 témoigne de la disparition de la dune au nord du wharf et devant les installations portuaires en construction (passerelle, entrepôts, etc.). Il n'a donc pas été possible d'extraire le pied de dune dans ces secteurs. De plus, de l'absence de contrastes des images de 1980 résulte l'impossibilité d'extraire localement la ligne des pleines mers; d'où l'importance du linéaire inexploité sur ces images.

La digitalisation du trait de côte aux trois dates retenues (1954, 1980 et 2005), a été répétée à plusieurs reprises de manière à estimer la marge d'erreur induite par l'opérateur lors de la numérisation à l'écran. En effet, comme l'ont indiqué plusieurs auteurs, le positionnement du trait côte au moment de la phase de digitalisation par un même opérateur peut être extrêmement variable dans le temps (Thieler et Danforth, 1994a; Durand, 2000). Cela dépend de la perception de l'information par la personne à un moment donné. Cela peut aussi relever de considérations techniques, comme le type d'ordinateur, de luminosité d'écran, etc., qui donnent à l'opérateur une visualisation différente des objets. Ainsi, la répétition des données acquises pour un même secteur a permis de calculer l'intervalle de variation des décalages en matière de positionnement (tableau 4). Globalement, on obtient une précision métrique pour la ligne des pleines mers sur des prises de vue de bonne qualité et à grande échelle comme celles de 2005. En revanche, l'incertitude est plus grande sur les images anciennes. La précision est moindre pour la ligne des pleines mers si le contraste de l'image est médiocre comme sur les clichés de 1980.

En additionnant les intervalles de variation pour chaque ligne, on obtient une précision de \pm 4,4 $\mathrm{m}$ pour le pied de dune et $\pm 3,6 \mathrm{~m}$ pour la ligne des pleines mers.

\section{Erreurs liées aux variations du niveau des pleines mers}

Une étude comparative fondée sur la photo-interprétation de la ligne des pleines mers requiert que les images soient acquises aux mêmes périodes dans des conditions de marée identiques ou proches afin de minimiser l'influence des fluctuations du niveau des pleines mers (Stafford et Langfelder 1971). Il est donc nécessaire d'évaluer les effets des variations du niveau de marée haute sur la position de la ligne des pleines mers, ce d'autant plus que les dates exactes et les conditions météorologiques de toutes les prises de vue utilisées sont, comme nous l'avons mentionné précédemment, inconnues. Quantifier précisément ces effets dans une zone comme Nouakchott est délicat, car d'un côté nous ne disposons pas de données concernant la pente de la plage au moment où les missions aériennes ont été réalisées et d'un autre côté il n'existe pas de marégraphe 


\begin{tabular}{|c|c|c|c|c|c|}
\hline $\begin{array}{c}\text { Date des prises } \\
\text { de vues }\end{array}$ & $\begin{array}{c}\text { Nature de la ligne } \\
\text { de référence }\end{array}$ & $\begin{array}{c}\text { Nombre de points } \\
\text { mesurés }\end{array}$ & $\begin{array}{c}\text { Moyenne des } \\
\text { écarts }(\mathrm{m})\end{array}$ & Écart type $(\mathrm{m})$ & $\begin{array}{c}\text { Intervalle de } \\
\text { variation des } \\
\text { décalages moyens } \\
(\mathrm{m})\end{array}$ \\
\hline 1954 & Pied de dune & 310 & 1,36 & 0,49 & $\pm 2,8$ \\
\hline 1980 & Pied de dune & 342 & 0,52 & 0,24 & $\pm 1,6$ \\
\cline { 2 - 6 } & $\begin{array}{c}\text { Ligne des pleines } \\
\text { mers }\end{array}$ & 338 & 0,85 & 0,26 & $\pm 2,3$ \\
\hline 2005 & $\begin{array}{c}\text { Ligne des pleines } \\
\text { mers }\end{array}$ & 320 & 0,53 & $0,3,3$ \\
\hline
\end{tabular}

Tableau 4 : Précision de la numérisation du trait de côte en fonction des lignes de référence et des photographies aériennes utilisées

Precision of the digitized shoreline according to the reference lines and aerial photographs used

sur le site. Néanmoins, les informations fournies par les études antérieures permettent d'avoir une idée sur les niveaux extrêmes des pleines mers et les pentes de la plage. Ainsi pour cartographier l'aléa de submersion à Nouakchott, le GRESARC (2006) avait calculé des niveaux de pleines mers extrêmes pour la période 1980-2006 à partir des données marégraphiques de Nouadhibou, Saint-Louis et Dakar qui sont les ports de référence les plus proches. L'estimation du niveau maximum, pour une pleine mer de vive-eau, était de 1,37 m EGM96 (2,22 m au-dessus du zéro des marées) et le niveau minimum (pour une pleine mer de morte-eau) était de 0,24 m EGM96 (1,29 m au-dessus du zéro des marées). Concernant les pentes de la plage, les profils réalisés par I. De Lanjamet (1988) indiquent des pentes allant de $2,5^{\circ}$ pour les plus faibles à $8,5^{\circ}$ pour les plus fortes. Ainsi, comme l'on montré Y.-F. Thomas et A. T. DIAW (1997), en fonction de la pente $(\theta)$ et de la hauteur d'eau (h), il est possible de calculer l'écart entre les niveaux de pleines mers maximale et minimale $(\Delta \mathrm{d})$ à partir de la formule suivante :

$$
\Delta \mathrm{d}=\mathrm{h} / \tan (\theta)
$$

Pour une pente de $2,5^{\circ}$, le décalage entre les lignes des pleines mers maximales et minimales sera de $21,62 \mathrm{~m}$ et pour une pente de $8,5^{\circ}$, l'écart sera de $6,24 \mathrm{~m}$ soit en moyenne $\pm 13,93 \mathrm{~m}$. En l'absence d'informations sur les conditions météorologiques et marégraphiques des missions aériennes, une valeur moyenne $\pm 14 \mathrm{~m}$ a été retenue comme marge d'incertitude approximative associée aux variations de la position de la ligne des pleines mers due aux oscillations du niveau des marées hautes.

\section{Définition de la marge d'erreur globale}

Compte tenu des incertitudes liées à la précision du GPS, au calage des images, à l'extraction du trait de côte et aux variations à court terme de la ligne des pleines mers, avant le calcul des taux d'évolution, une marge d'erreur globale a été définie, en d'autres termes, des seuils à partir desquels on considère qu'il y a une avancée ou un recul du trait de côte. Cette marge d'erreur est le résultat de la somme de l'ensemble des erreurs qui ont été estimées précédemment. Ainsi pour la période 1954-1980, la marge d'erreur globale a été établie à $\pm 10,4 \mathrm{~m}$ et à \pm 23,60 m pour la période 1980-2005 (tableau 5). 


\begin{tabular}{|c|c|c|c|c|}
\hline Période d'étude & Ligne de référence & \multicolumn{2}{|c|}{ Source d'incertitude } & $\begin{array}{l}\text { Estimation de } \\
\text { l'erreur }(\mathrm{m})\end{array}$ \\
\hline \multirow{5}{*}{ 1954-1980 } & \multirow{5}{*}{ Pied de dune } & \multicolumn{2}{|c|}{ Récepteur GPS } & \pm 4 \\
\hline & & \multicolumn{2}{|c|}{ Calage des images (Erreur RMS) } & \pm 2 \\
\hline & & \multirow{2}{*}{$\begin{array}{l}\text { Digitalisation du } \\
\text { pied de dune }\end{array}$} & 1954 & $\pm 2,8$ \\
\hline & & & 1980 & $\pm 1,6$ \\
\hline & & \multicolumn{2}{|c|}{ TOTAL (Marge d'erreur) } & $\pm 10,4$ \\
\hline \multirow{6}{*}{$1980-2005$} & \multirow{6}{*}{$\begin{array}{l}\text { Ligne des pleines } \\
\text { mers }\end{array}$} & \multicolumn{2}{|c|}{ Récepteur GPS } & \pm 4 \\
\hline & & \multicolumn{2}{|c|}{ Calage des images (Erreur RMS) } & \pm 2 \\
\hline & & \multirow{2}{*}{$\begin{array}{r}\text { Digitalisation de la } \\
\text { ligne des pleines mers }\end{array}$} & 1980 & $\pm 2,3$ \\
\hline & & & 2005 & $\pm 1,3$ \\
\hline & & \multicolumn{2}{|c|}{$\begin{array}{l}\text { Variation à court terme de la position de la } \\
\text { ligne des pleines mers }\end{array}$} & \pm 14 \\
\hline & & \multicolumn{2}{|c|}{ TOTAL (Marge d'erreur) } & $\pm 23,60$ \\
\hline
\end{tabular}

Tableau 5 : Estimation de la marge d'erreur globale

Estimation of the total error margin

\section{Mesure de la cinématique du TRAit de CÔTE}

Les évolutions ont été mesurées automatiquement grâce au programme DSAS (Digital Shoreline Analysis System) (Thieler et Danforth, 1994b) suivant des transects perpendiculaires aux lignes à comparer. Ces transects terre/mer, espacés de $10 \mathrm{~m}$, soit dans notre cas plus d'un millier pour chacune des deux périodes étudiées, sont générés à partir d'une ligne de base (fig. 2). Le DSAS mesure les distances entre les points d'intersection des transects et des traits de côte, calcule les taux d'évolution le long de chaque transect et restitue les résultats sous forme de tables attributaires. L'estimation de la cinématique du trait de côte et la cartographie des taux d'évolution sont basées sur l'analyse de ces tables attributaires. Etant donné que nous disposions de deux positions du pied de dune (1954 et 1980) et deux positions de la ligne des pleines mers (1980 et 2005), nous avons choisi l'indice EPR (End point rate) proposé par le DSAS. Cet indice est calculé selon la méthode des points extrêmes qui utilise la position la plus ancienne et la plus récente de chaque ligne de référence (Dolan et al., 1991). La distance entre ces deux positions le long de chaque transect est divisée par le temps écoulé pour obtenir la vitesse d'évolution au niveau de ce point. Les zones qui présentaient une tendance positive ou négative plus ou moins homogène ont été regroupées en secteurs. Par la suite, les mesures de distances et les taux d'évolution concernant ces segments de côte ont été moyennés pour cartographier l'évolution diachronique du pied de dune et de la ligne des pleines mers (fig. 3 et 4 ).

\section{Résultats}

La comparaison des traits de côte de 1954 et de 1980 témoigne d'une érosion du littoral intervenue entre ces deux dates, en accord avec les conclusions de J. P. Barusseau (1985). Le taux moyen de recul est de $-0,76 \mathrm{~m} / \mathrm{an}$ si on tient compte de toutes les mesures. Si on exclut les valeurs situées dans la marge d'incertitude soient 16,25\% des transects, le taux moyen de recul passe à $-0,83$ m/an. Ainsi, les résultats peuvent être légèrement influencés par la marge d'erreur, particulièrement dans les secteurs à faible évolution comme au nord de l'hôtel Ahmedy (fig. 3) où 62,79\% des mesures sont inférieures ou égales à $\pm 10,4 \mathrm{~m}$. Cette influence est négligeable pour tous les autres segments qui présentent des valeurs de recul largement supérieures à la marge d'erreur. 
Figure 2 : Exemple de transects et d'une ligne de base, créés par le DSAS pour mesurer l'évolution du trait de côte entre 1954 et 1980

Sample of transects and baseline edited by the DSAS program to compute shoreline change rates from 1954 to 1980

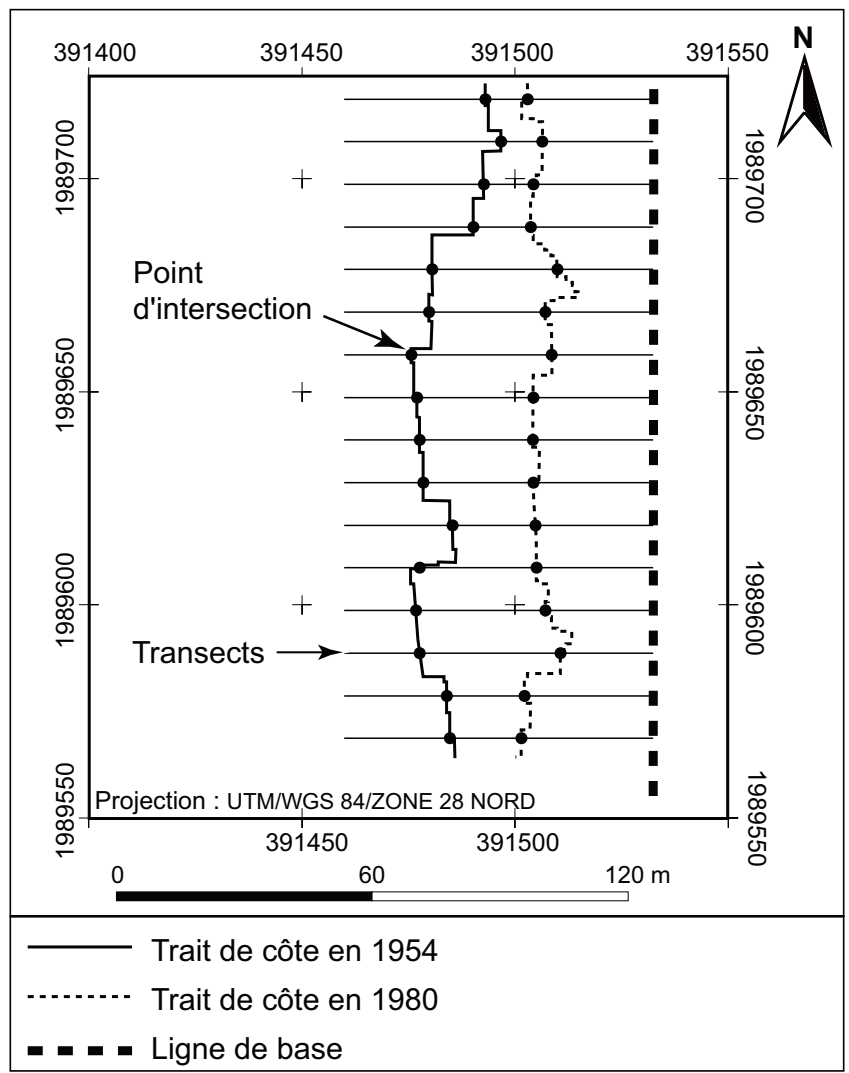

Du point de vue sectoriel (fig. 3), entre 1954 et 1980, la côte présente une forme légèrement concave avec des vitesses d'érosion variables. L'extrémité septentrionale de cette zone concave semble reculer beaucoup plus lentement que les autres secteurs avec $-0,50 \mathrm{~m} / \mathrm{an}$ au nord de l'hôtel Ahmedy. Les plus forts taux d'érosion sont localisés de part et d'autre du wharf soit - $0,81 \mathrm{~m} / \mathrm{an}$ au nord et $-0,90 \mathrm{~m} / \mathrm{an}$ au sud. Cette évolution s'expliquerait par des causes naturelles en l'absence de toute infrastructure ou aménagement susceptible de perturber la dynamique du rivage. En effet, en 1954 la côte apparaît vierge de toute occupation humaine alors qu'en 1980, les quelques infrastructures présentes sur le littoral se limitent au wharf, à l'hôtel Ahmedy et aux installations portuaires en construction.

Pour la période suivante (1980-2005), hormis les environs de l'hôtel Ahmedy où 5,34 \% des mesures tombent dans la marge d'incertitude, les évolutions mesurées sont largement supérieures à \pm 23,6 m. La construction d'un mur pour bloquer les sédiments en dessous de la passerelle du port et d'un épi quelques centaines de mètres plus au sud a divisé le littoral en deux parties (fig. 4) : une zone septentrionale en progradation et une zone méridionale en érosion. Dans la zone au nord de la passerelle, la vitesse d'avancée du trait de côte varie de 1,85 à 4,62 m/an entre l'hôtel Ahmedy et le wharf. Au sud du wharf, la progradation du linéaire côtier augmente graduellement en direction du port en passant de 6,44 m/an à proximité de l'épave du bateau Walter à 32,94 m/an immédiatement au nord de la passerelle. Cette progradation du trait de côte approximativement sur une longueur de $8 \mathrm{~km}$ se manifeste sur le terrain en avant de l'ancienne dune par une large banquette prédunaire colonisée par une végétation psammophile dominée par Zygophyllum waterlotii. 


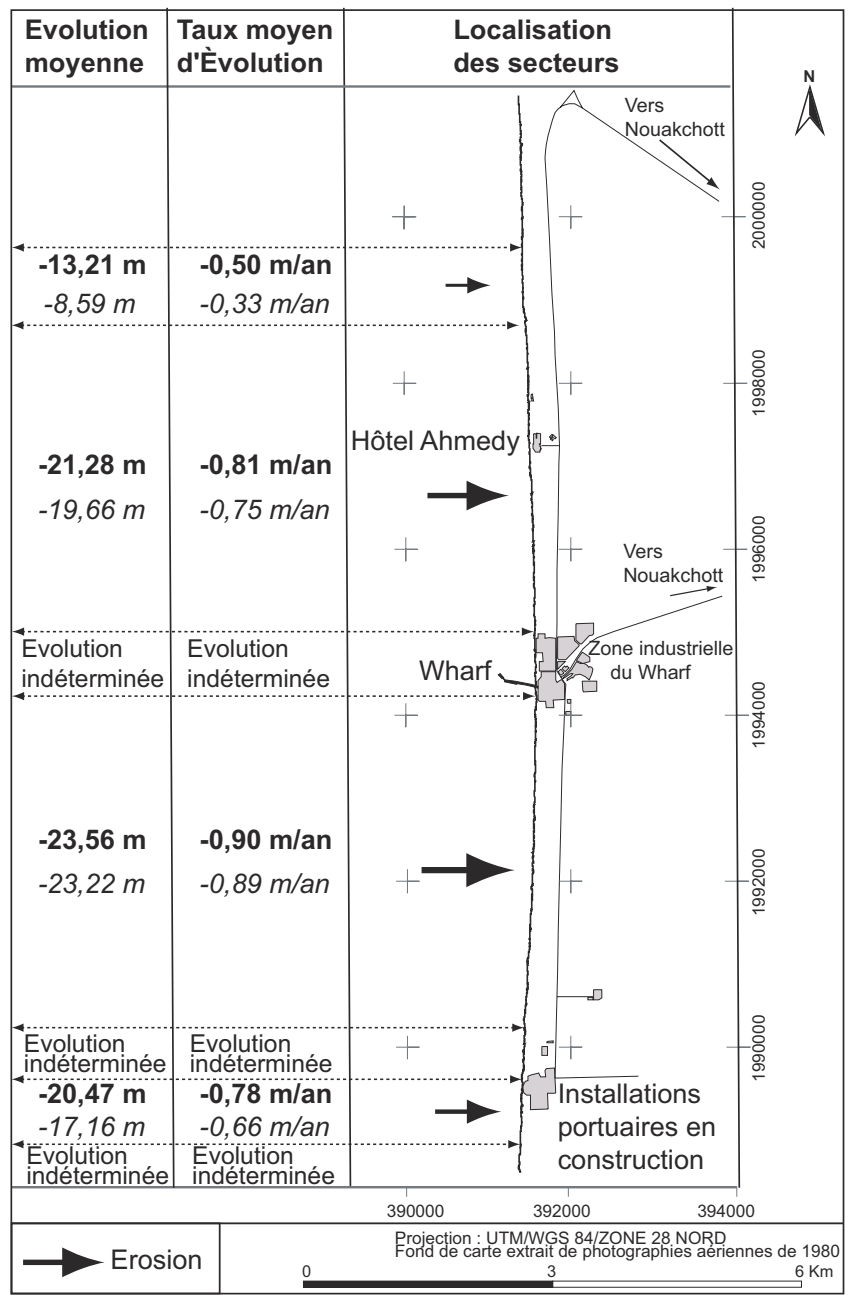

Figure 3 : Évolution sectorielle du pied de dune dans la zone étudiée de 1954 à 1980. (En gras, les taux d'évolution hors marge d'erreur et en italiques les taux d'évolution avec la marge d'erreur)

Sector-based evolution of the dune foot position in the studied area from 1954 to 1980 . (In bold, rates of change without the error margin; in italics rates of change with the error margin)

Au sud de la passerelle, l'épi construit entre mai 1990 et juillet 1991, a créé une zone d'accumulation longue de presque $700 \mathrm{~m}$ où le trait de côte a progressé à un taux moyen de 4,86 m/an de 1980 à 2005. A contrario, durant cette période le recul a été très important au sud de l'épi. Il est de l'ordre de $-526,88 \mathrm{~m}$, soit -21,07 m/an le long des $2 \mathrm{~km}$ de côte couverts par les prises de vue de 2005 .

\section{Discussion et conclusion}

L'utilisation de la photo-interprétation pour l'étude diachronique de la cinématique d'un littoral comme Nouakchott se heurte essentiellement à la qualité des données photographiques disponibles et au choix d'une ligne de référence repérable sur les clichés multidates. Le pied de dune lorsqu'il existe semble être un bon marqueur géomorphologique pour retracer la position du trait de côte, mais il n'est pas détectable sur les photographies postérieures aux années 1980 à cause de la dégradation du cordon dunaire. Quant à la ligne des pleines mers, les variations de ses positions sur le court terme peuvent être très importantes même si elles restent inférieures à l'ampleur des évolutions engendrées par le port. Son utilisation comme indicateur nécessite au minimum des évolutions de plusieurs dizaines de mètres pour traduire des tendances évolutives fiables. 
Figure 4 : Évolution sectorielle de la ligne des pleines mers entre l'hôtel Ahmedy et le port de Nouakchott de 1980 à 2005 (en gras, les taux d'évolution hors marge d'erreur et en italiques les taux d'évolution avec la marge d'erreur) Sector-based evolution of the high water line position between Ahmedy hotel and Nouakchott harbour from 1980 to 2005 (in bold, rates of change without the error margin; in italics rates of change with the error margin)

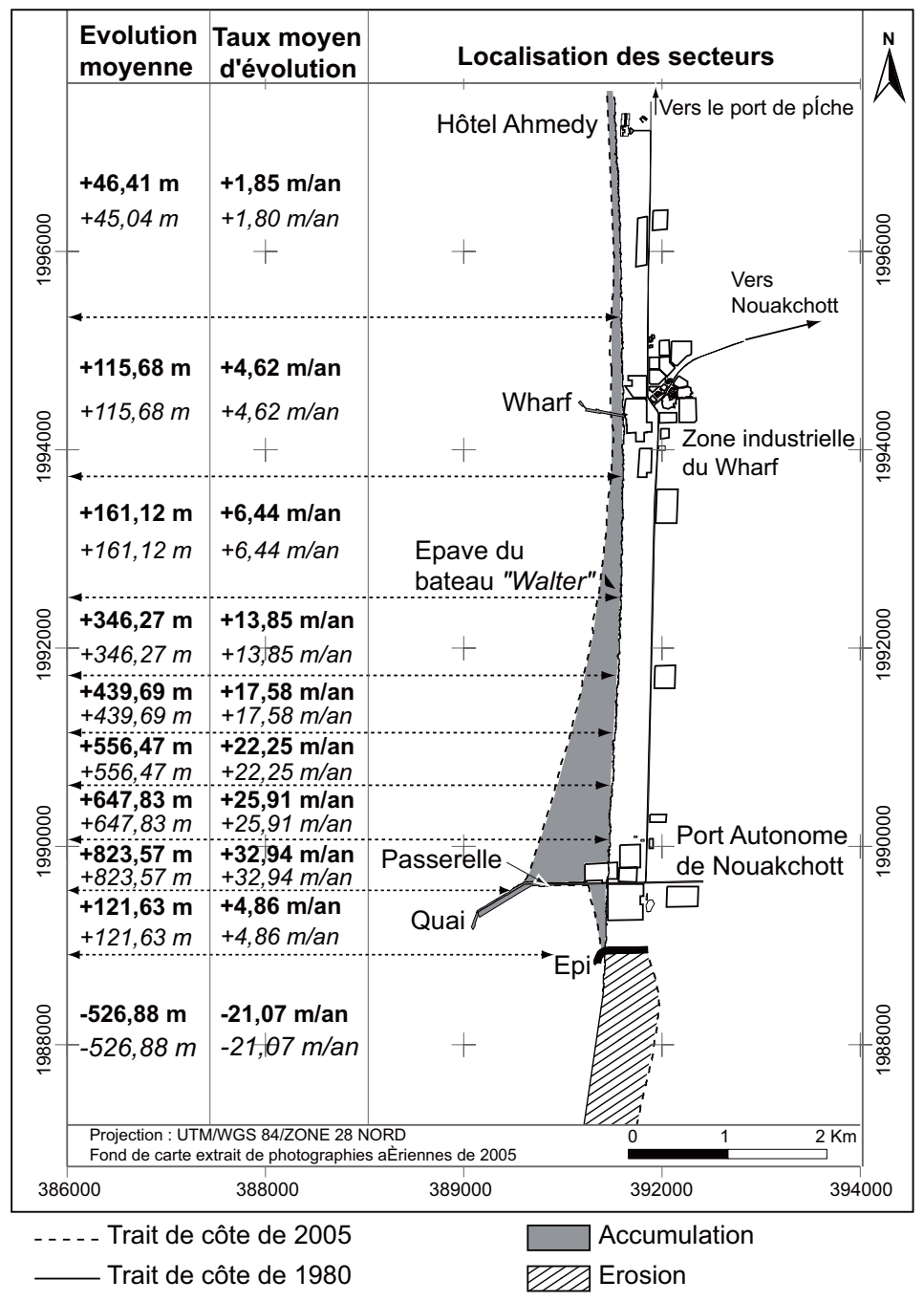

Concernant la cinématique littorale de 1954 à 2005, malgré les limites inhérentes à notre approche méthodologique, le suivi de la position du pied de dune et de la ligne des pleines mers a permis de mesurer l'évolution du trait de côte à une échelle mi-séculaire (1954-2005). Ainsi entre 1954 et 1980, le littoral était en érosion naturelle à un rythme moyen de l'ordre de 0,83 m/an du nord de l'hôtel Ahmedy jusqu'au port. Depuis le début des années 1980, les aménagements portuaires ont perturbé cette tendance et induit des évolutions majeures faisant de Nouakchott un cas extrême de dynamique littorale où les forçages anthropiques sont prépondérants sur les forçages naturels (Marico, 1996; Philippon, 1999; Ould El Moustapha, 2000). Les taux d'évolution que nous avons calculés aux abords du port pour la période 1980-2005 sont cohérents avec les tendances relevées dans la littérature. Toutefois, les vitesses d'évolution mesurées diffèrent légèrement de celles estimées par A. Ould El Moustapha et al. (2007) (+32,7 m/an et -25 m/an) sur une échelle temporelle sensiblement égale à la nôtre (1980-2002). Ces divergences peuvent être expliquées par la diversité des lignes de référence, des sources de données et des approches méthodologiques utilisées. 
Des recherches sur la cinématique du trait de côte sont actuellement en cours dans d'autres sites ouest-africains et à l'échelle régionale. Elles contribueront à décrire les tendances des changements à long terme dans le but d'analyser les principaux facteurs déterminant l'évolution de la zone côtière et d'en déduire des scénarios plausibles.

\section{Bibliographie}

Anders (F. J.), Byrnes (M. R.), 1991. - "Accuracy of shoreline change rates as determined from map and aerial photographs", Shore and Beach, vol. 59, n 1, p. 17-26.

AZANdossessi (A.), 1988. - Évolution de la ligne de rivage au sud du port de l'Amitié, Nouakchott, mémoire de fin d'études, École Normale Supérieure de Nouakchott, 33 p.

BAI (Z.), LI (D.), LI (S.) 1997. - Report on mathematical model test research of sediment alluvial and wave shielding for Nouakchott fishery terminal project in Mauritania. Middle Report $N^{\circ} 1$ - Synthetical analysis on basic hydrologic data in engineering sea region and verification on sediment transport by mathematical model test in friendship harbor, Tianjin Port Engineering Institute, Tianjin (Chine), n.p.

Barusseau (J.-P.), 1985. - Évolution de la ligne de rivage en République Islamique de Mauritanie, Paris, rapport UNESCO, Division des Sciences de la Mer, 104 p.

Bind (E. C. F.), 1985 - Coastline changes. A global review, Chichester, John Wiley \& Sons, A Wiley - Interscience publication, $219 \mathrm{p}$.

Boak (E. H.), Turner (I. L.), 2005. - "Shoreline definition and detection: A review", Journal of Coastal Research, vol. 21, n 4, p. 688-703.

Crowell (M.), Leatherman (S. P.), Buckley (M. K.), 1991. - "Historical shoreline change : Error analysis and mapping accuracy", Journal of Coastal Research, vol. 7, n 3, p. 839-852.

Denouck (A.), 2006. - Morphodynamique des plages sableuses de la mer d'Iroise (Finistère), Brest, thèse de doctorat en géographie physique, Université de Bretagne Occidentale, 262 p.

De Lanjamet (I.), 1988. - La grande plage mauritanienne, Nouakchott, Centre Culturel Français Antoinede-Saint-Exupéry, coll. «Connaissance de la Mauritanie », 93 p.

Dolan (R.), Fenster (M. S.), Holme (S. J.), 1991. - "Temporal analysis of shoreline recession and accretion”, Journal of Coastal Research, vol. 7, n³ 3, p. 723-744.

DuRAND (P.), 1998 - «Cinématique d'un littoral sableux à partir de photographies aériennes et de cartes topographiques. Exemple du littoral d'Argelès-Plage à Saint-Cyprien (Roussillon - France) », Géomorphologie : relief, processus, environnement, $\mathrm{n}^{\circ} 2$, p. 155-166.

—, 2000 - «Approche méthodologique pour l'analyse des littoraux sableux par photo - interprétation. Exemple des plages situées entre l'Aude et l'Hérault (Languedoc, France) », Photo-Interprétation, nº 1-2, p. 3-18.

Gaillot (S.), Chaverot (S.) 2001 - « Méthode d'étude des littoraux à faible évolution. Cas du delta du Golo (Corse) et du littoral du Touquet (Pas de calais) en France », Géomorphologie : relief, processus, environnement, $\mathrm{n}^{\circ} 1$, p. 47-54.

GresarC (Groupe de Recherche sur les Environnements Sédimentaires Aménagés et les Risques Côtiers), 2006. - Cartographie des risques littoraux de Nouakchott, Université de Caen (Unité de morphodynamique continentale et côtière), Caen, 22 p. + Annexes, n.p.

Ibe (A. C.), Quelennec (R. E.), 1989 - "Methodology for assessment and control of coastal erosion in West and Central Africa", UNEP Regional Seas Reports and Studies, Nairobi, n 107, 107 p.

MARico (D.), 1996. - Contribution à l'étude géomorphologique des côtes mauritaniennes : du Cap Timiris à Ndiago, Tunis, thèse de doctorat de troisième cycle (Diplôme de Recherches Approfondies : DRA) - Option Géographie physique, Université des Lettres, des Arts et des Sciences Humaines - Tunis I, $227 \mathrm{p}$.

Moore (L. J.), 2000. - "Shoreline mapping techniques", Journal of Coastal Research, vol. 16, n 1, p. 111-124.

Ould el Ghassem (D.), 1987. - Le littoral mauritanien au sud de Nouakchott. Évolution morphologique au voisinage du Port de l'Amitié, Nouakchott, mémoire de fin d'études, École Normale Supérieure de Nouakchott, 51 p. 
Ould el MoustapHA (A.), 2000. - Influence d'un ouvrage portuaire sur l'équilibre d'un littoral soumis à un fort transit sédimentaire. L'exemple du port de Nouakchott (Mauritanie), Caen, thèse de doctorat d'université, Université de Caen/Basse Normandie, 162 p.

Ould el Moustapha (A.), Levoy (F.), Monfort (O.), Koutitonsky (V. G.), 2007. - "A numerical forecast of shoreline evolution after harbour construction in Nouakchott, Mauritania”, Journal of Coastal Research, vol. 23, nº 6, p. 1409-1417.

Ould Eleya (A. B.), 1994. - « Le port et les modifications du profil du rivage », dans Aménagement du littoral mauritanien. Actes de l'Atelier National sur le Littoral Mauritanien. Nouakchott du (samedi) 26 au (mardi) 29 novembre 1994, Nouakchott, République Islamique de Mauritanie/ministère de l'Intérieur, des Postes et Télécommunications/Direction de l'Aménagement du Territoire et de l'Action Régionale, publié avec le concours de l'UICN et de AFRICA 70, p. 99-103.

PhilipPon (S.), 1999. - La côte de Nouakchott (Mauritanie) : une évolution naturelle et anthropique, Paris, mémoire de maîtrise de géographie, Université Paris 1 Panthéon - Sorbonne, 165 p.

Quensiere (J.), Diaw (A. T.), Ould el Moustapha Senhoury (A.), Charles-Dominique (E.), 2006. - « Un réseau ouest - africain de recherche sur la gestion durable des zones côtières : le projet "Bilan Prospectif", dans Symoens (J.-J.) (dir.), Les écosystèmes côtiers de l'Afrique de l'Ouest. Diversité biologique - Ressources - Conservation, Bruxelles, SBWOA, p. 159-176

Robin (M.), 2002 - « Télédétection et modélisation du trait de côte et de sa cinématique » dans BARONYelles (N.), Goeldner-Gionella (L.), Velut (S.) (dir.), Le littoral, regards, pratiques et savoirs; études offertes à Fernand Verger, Paris, éditions Rue d'Ulm, Presses universitaires de l'École Normale Supérieure, p. 95-115.

Shoshany (M.), Degani (A.) (1992) - "Shoreline detection by digital image processing of aerial photography", Journal of Coastal Research, vol. 8, n 1, p. 29-34.

Stafford (D. B.), Langfelder (J.), 1971. - «Air photo survey of coastal erosion », Photogrammetric Engineering, vol. 37, p. 565-575.

Thieler (R. E.), Danforth (W. W.), 1994a. - "Historical shoreline mapping I: Improving techniques and reducing positioning errors", Journal of Coastal Research, vol. 10, n 3, p. 549-563.

Thieler (E. R.), Danforth (W. W.), 1994b. - "Historical shoreline mapping II: Application of the digital shoreline mapping and analysis systems (DSMS/DSAS) to shoreline change mapping in Puerto Rico", Journal of Coastal Research, vol. 10, n³ 3, p. 600-620.

Thomas (Y.-F.), Diaw (A. T.), 1997. - « Suivi (1984-1993) de la rupture de la flèche de Sangomar, estuaire du Saloum, Sénégal », Photo-Interprétation, n³-4, p. 199-204.

Wu (W.), 2003. - Application de la géomatique au suivi de la dynamique environnementale en zone aride. Exemple de la région de Nouakchott en Mauritanie, du Ningxia nord et du Shaanxi nord en Chine du nord-onest. Paris, thèse de doctorat, Université Paris 1 - Panthéon-Sorbonne/École Pratique des Hautes Études, 217 p.

\section{Remerciements}

Cet article s'inscrit dans le cadre d'une recherche doctorale financée par la Fondation Internationale du Banc d'Arguin (FIBA) à travers le projet «Bilan prospectif », composante recherche du Programme Régional de Conservation de la Zone Côtière et Maritime en Afrique de l'Ouest (PRCM).

Cet article a été reçu le 6 mars 2008 et définitivement accepté le 2 octobre 2008. 
